\title{
Superferromagnetism and Domain-Wall Topologies in Artificial "Pinwheel" Spin Ice
}

Yue Li, ${ }^{\dagger}$ Gary W. Paterson, ${ }^{*}{ }^{\dagger}$ G Gavin M. Macauley, ${ }^{\dagger}$ Fabio S. Nascimento, ${ }^{\dagger}$ Ciaran Ferguson, ${ }^{\dagger}$ Sophie A. Morley, ${ }^{\nabla, \S}$ Mark C. Rosamond, ${ }^{\|}$Edmund H. Linfield, $"$ Donald A. MacLaren, ${ }^{\dagger}$ Rair Macêdo, ${ }^{\dagger}$ Christopher H. Marrows, ${ }^{\nabla}$ Stephen McVitie, ${ }^{* \dagger},{ }^{\dagger}$ and Robert L. Stamps ${ }^{*}, \dagger, \perp$

${ }^{\dagger}$ SUPA, School of Physics and Astronomy, University of Glasgow, Glasgow G12 8QQ United Kingdom

${ }^{\ddagger}$ Departamento de Física, Universidade Federal de Viçosa, Viçosa 36570-900, Minas Gerais, Brazil

${ }^{\nabla}$ School of Physics and Astronomy, University of Leeds, Leeds LS2 9JT, United Kingdom

${ }^{\S}$ Department of Physics, University of California, Santa Cruz, California 95064, United States

"School of Electronic and Electrical Engineering, University of Leeds, Leeds LS2 9JT, United Kingdom

${ }^{\perp}$ Department of Physics and Astronomy, University of Manitoba, Manitoba R3T 2N2, Canada

\section{Supporting Information}

ABSTRACT: For over ten years, arrays of interacting singledomain nanomagnets, referred to as artificial spin ices, have been engineered with the aim to study frustration in model spin systems. Here, we use Fresnel imaging to study the reversal process in "pinwheel" artificial spin ice, a modified square ASI structure obtained by rotating each island by some angle about its midpoint. Our results demonstrate that a simple $45^{\circ}$ rotation changes the magnetic ordering from antiferromagnetic to ferromagnetic, creating a superferromagnet which exhibits mesoscopic domain growth mediated by domain wall nucleation and coherent domain propagation. We observe several domainwall configurations, most of which are direct analogues to those
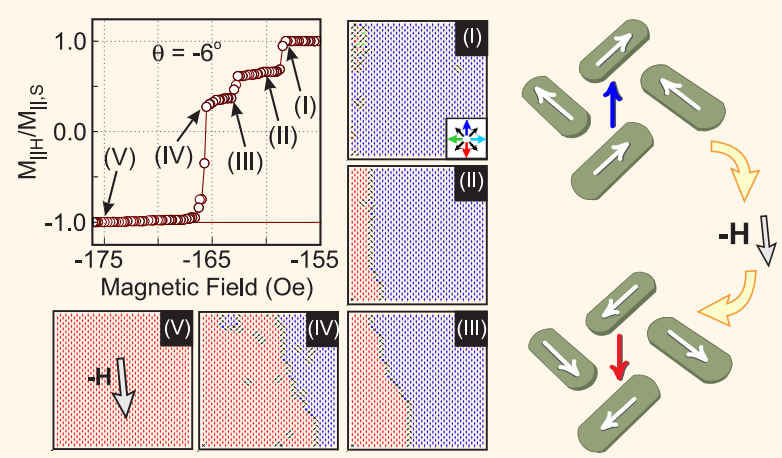
seen in continuous ferromagnetic films. However, charged walls also appear due to the geometric constraints of the system. Changing the orientation of the external magnetic field allows control of the nature of the spin reversal with the emergence of either one- or two-dimensional avalanches. This property of pinwheel ASI could be employed to tune devices based on magnetotransport phenomena such as Hall circuits.

KEYWORDS: artificial spin ice, superferromagnetism, magnetization process, mesoscopic domain wall, Lorentz transmission electron microscopy

A

rtificial spin ice (ASI) systems have been used not only as a route to new physical phenomena but also to gain insight into fundamental physics. Such capabilities are only possible because these structures are able to emulate the behavior of assemblies of the individual spins in atomic systems. This is done by controlling the shape and size of each nanoelement to ensure that they behave as single-domain magnets. One of the most appealing and perhaps well-known aspects of ASI systems is their capability to display geometrical frustration. This magnetic topological frustration gives rise to interesting properties, ${ }^{1-12}$ such as monopole-like defects ${ }^{5,11-15}$ and multi-fold ground state degeneracy. $1,2,9,16$

The classic ASI tiling, that of square ice, has a well-known long-range antiferromagnetic ground state arising from its twofold degenerate two-in-two-out spin configuration of each vertex. $^{7,17,18}$ This structure, which obeys the so-called ice rule $^{19}$ and possesses four well-defined vertex energies, was initially investigated by Wang et al. ${ }^{1}$ Their work ignited great interest in not only square ASI, but also in several other ASI arrangements. In particular, Morrison et al. ${ }^{20}$ pointed out the importance of vertex interactions and their dependence on geometry. In fact, a simple modification of the square ASI system provided a recent example of emergent dynamics: the "pinwheel" ice. ${ }^{21,22}$ The pinwheel geometry is obtained by rotating each island in square ASI around its center. Gliga et al. have found that thermal relaxation in this system behaves as if it obeyed an intrinsic chirality. ${ }^{21}$ Frustration in pinwheel ASI is theoretically predicted to be markedly different than that in square ice in that the energies of the different pinwheel units

Received: November 21, 2018

Accepted: December 27, 2018

Published: December 27, 2018 
are found to be nearly degenerate, whereas the energy levels of square ice vertices are well separated. ${ }^{22}$

In this work, we use Lorentz transmission electron microscopy (LTEM) $)^{23}$ to directly visualize the magnetization reversal process in a pinwheel ASI array in the presence of a static externally applied magnetic field. Under such conditions, the system behaves as a superferromagnet, that is, an ensemble of macrospins with collective ferromagnetic behavior. ${ }^{24}$ Our superferromagnet has coherent domain growth and shrinking as opposed to the chain avalanche reversal seen in square ASI. ${ }^{25,26}$ The different magnetic domains seen in pinwheel ASI are separated by domain walls, some of which behave much like the classical ferromagnetic Néel domain walls in continuous films. However, in pinwheel ASI, different types of charged domain walls are also observed, and the magnetic charge ordering of these walls is dependent on the magnetization alignment of the neighboring domains. The behavior of these walls and domains is significantly affected by the field orientation, which is also investigated. These properties of pinwheel ASI offer a possible avenue to design functional materials exploiting the emergent magnetic spin textures and controllable reversal dimensionality of the system.

\section{RESULTS}

Array edges for pinwheel ASI arrays are typically either diamond or lucky-knot designs, ${ }^{22}$ where the array termination edges lie at either $45^{\circ}$ or $90^{\circ}$ to an island long axis, respectively. Here, we investigate the behavior of a diamondedge permalloy $\left(\mathrm{Ni}_{80} \mathrm{Fe}_{20}\right)$ pinwheel array of two interleaved collinear $25 \times 25$ sublattices as shown in the in-focus TEM image in the upper half of Figure 1. For this edge type, the element centers define a diamond geometry ${ }^{22}$ (as shown in Figure 1, top left inset). Each individual nanomagnetic island is $10 \mathrm{~nm}$ thick, $470 \mathrm{~nm}$ long, and $170 \mathrm{~nm}$ wide, with a center-tocenter separation between nearest-neighbor islands of $420 \mathrm{~nm}$, as shown the top right inset to Figure 1.

Ising Hysteresis Behavior. In order to characterize the behavior of the pinwheel ASI, we first look at the behavior of the Ising net magnetization of the individual pinwheel units, where each unit is formed by the four nearest-neighbor islands. This is done by examining the defocused Fresnel LTEM images recorded during a reversal, an example of which is shown in the bottom half of Figure 1. Magnetic contrast arises through deflection of the electron beam by the induction from the magnetization of each island. Since the magnetization lies along the island's long axis, a thin dark edge will be seen on one side and a broad dark edge on the other, with the direction dependent on the orientation of the moment. From this, the magnetization direction of each unit can be directly measured through its magnetic contrast, as shown in the inset of Figure 1 (details of the image processing methodology used to do this are given in the Methods section and Supporting Information). In this way, it is then possible to follow the moment orientations throughout the entire array as a function of the external field. Example Ising hysteresis loops extracted from these orientations are given in Figure $2 a-c$ for various field angles with respect to the array edges, as defined in Figure 1. Although the coercivity for each field angle is slightly different, the general behavior is similar. Note that when the external static field lies parallel to the $y$-axis $(\theta=0)$, the component of the externally applied field along the easy-axis of each of the pinwheel islands is the same. The measured coercive field, $H_{\mathrm{C}}$, is in agreement with that calculated using the field protocols

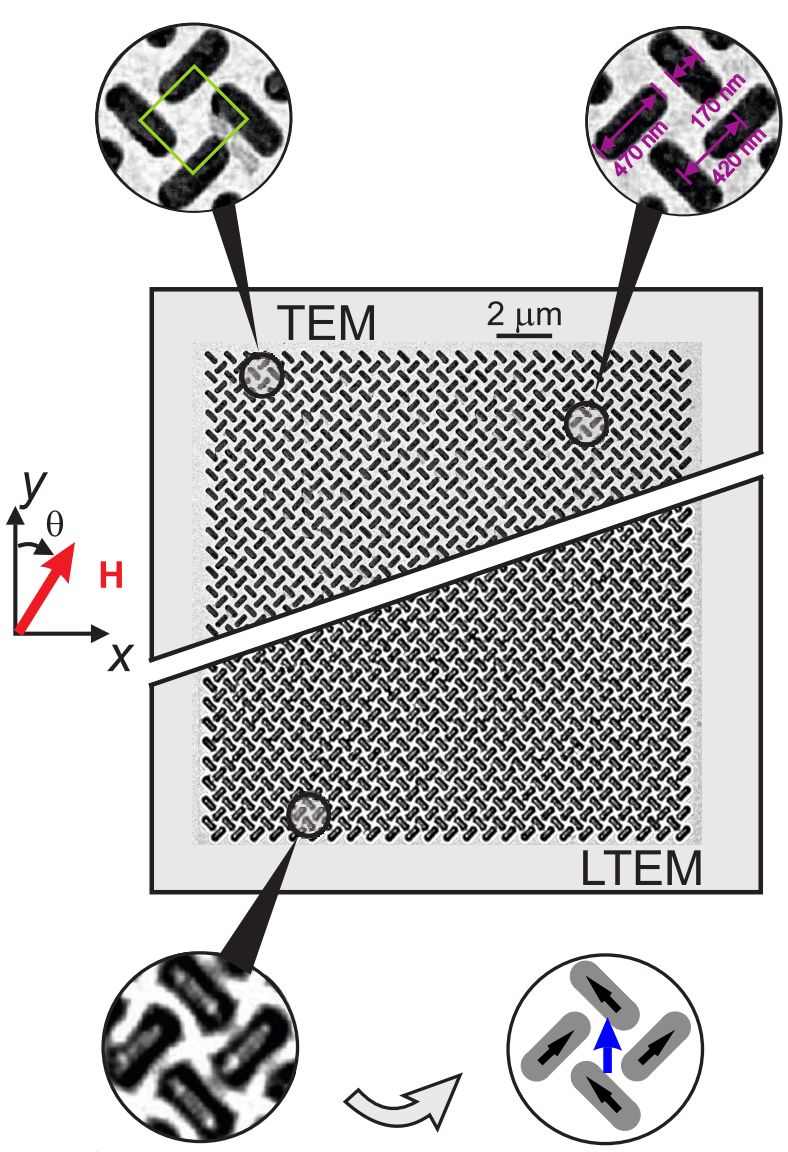

Figure 1. Example of experimental in-focus TEM and LTEM images of an artificial pinwheel spin ice array. The top-left part shows an in-focus TEM image of a pinwheel ASI array composed of two interleaved $25 \times 25$ sublattices. To the left, the field direction defined with respect to the array edges is shown. The top-left inset is the zoomed-in image of a diamond pinwheel unit, and the top-right inset shows the in-plane dimension of each island and the center-to-center distance between nearest-neighbor islands. The bottom-right part of the array is an LTEM image of the same pinwheel array with an enlarged view of a single unit (the bottom-left inset). In this zoom-in pinwheel unit Fresnel image, a broader dark edge on the right-hand side of each nanomagnet allows us to assign the direction of magnetization, as schematically shown in the bottom-right inset.

outlined in Supporting Information (see Figure S6). However, it is worth noting that despite extensive efforts to replicate the precise details of the magnetization, this model does not adequately capture the richness of the behavior discussed in the following sections.

For an array of entirely uncoupled islands, the easy anisotropy axis should lie at $\theta=0$. Inter-island interactions may modify this angle in a real system, so it is important to characterize it experimentally. We can determine the anisotropy axis of our array by examining the net magnetization component perpendicular to the field. This component is small at low-angles of applied field and, in fact, shows an interesting dependence on the applied field angle. This can easily be seen if the $x$ - and $y$-components of the net magnetization along the array axes are plotted in polar coordinates. In Figure $2 \mathrm{~d}-\mathrm{f}$, we re-plot in this way the data 

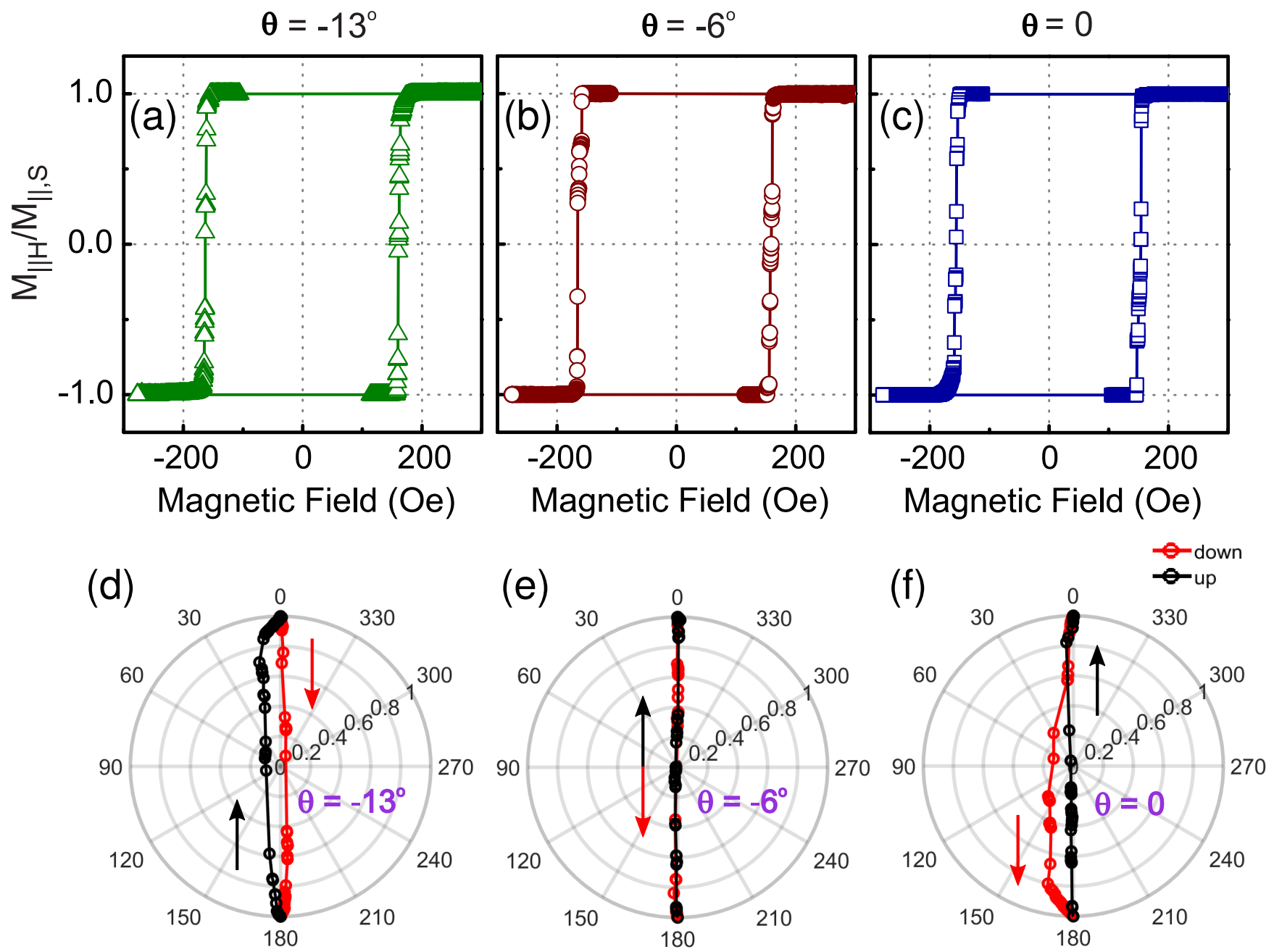

Figure 2. $(a-c)$ Normalized component of the array net magnetization aligned with the field, $M_{\|}$, during a field sweep for $(a) \theta=-13^{\circ},(b) \theta$ $=-6^{\circ}$, and $(\mathrm{c}) \theta=0 .(\mathrm{d}-\mathrm{f})$ Normalized net moment of the entire array displayed in polar coordinates, with the field aligned at the same angles as those in $(\mathrm{a}-\mathrm{c})$. The down $(\mathrm{H}<0)$ and up branches are indicated by the symbol color (red and black) and arrow directions.

of Figure $2 a-c\left(-13^{\circ},-6^{\circ}\right.$, and 0$)$. In order to understand the meaning of these plots, it is useful to think of the pinwheel array as two interpenetating sublattices of collinear islands ${ }^{21}$ (see details in Figure S7b). With the external field applied parallel to the easy anisotropy axis of the array, the reversal of each sublattice happens simultaneously, causing the polar hysteresis loop to collapse, and the reversal should be described by overlapping lines to and from $0^{\circ}$ and $180^{\circ}$. For the pinwheel array, this occurs at $\theta=-6^{\circ}$ as shown in Figure 2e. When the field is misaligned with the easy axis, the magnetization rotates with a sense of direction that reflects the sign of the angle, $\theta$. For example, in Figure $2 \mathrm{~d}$ for $\theta=-13^{\circ}$, the moment rotates clockwise, whereas in Figure $2 \mathrm{f}$ for $\theta=0$, the moment rotates anticlockwise.

Using the width of the polar hysteresis loop as a measure of the angle between the applied field and anisotropy axes, we estimate that the anisotropy axis lies at $-5.7^{\circ} \pm 1.4^{\circ}$ to the array edge (see Figure S8 for further details). Careful measurement of in-focus TEM images of an untilted array confirms that the angles between the sublattices and with respect to the array edges in the realized array are accurate to within $\pm 0.6^{\circ}$. While this interesting result deserves further investigation, it is beyond the scope of this work, and, other than the angle offset, we do not expect it to dramatically affect the reversal process which will be discussed in the following sections.

Magnetization Reversal and Domain Formation. The hysteresis loops shown in Figure $2 a-c$, constructed using the component of magnetization parallel to the field, $M_{\|}$, suggests a ferromagnetic ordering of the array. Due to the mesocopic scale inherent to ASI, we can examine the Ising magnetization of individual islands as a function of the externally applied field. As is commonly done in ASI, for the reminder of this work we will consider the array as a system of four-island units, as shown in the insets of Figure 1. Here and elsewhere, we adopt the unit type names from square ASI vertices ${ }^{27}$ (see detailes in Figure S9). So far, we have only discussed the ferromagnetic behavior associated with small angles of the externally applied static field. We find that the magnetic ordering and reversal behavior at high angles of applied field are markedly different.

Reversal at Low Applied Field Angles. In Figure 3, we show examples of field-driven evolution of the four island unit magnetization configuration composing an entire pinwheel array in the vicinity of coercive field. The colors represent the unit magnetization directions as defined in the legend. We show snapshots at different field angles in Figure $3 a, b$ at the field magnitudes, marked in the hysteresis loops from (I) to (V) (further details of the $-6^{\circ}$ data can be seen in Video S1 of 

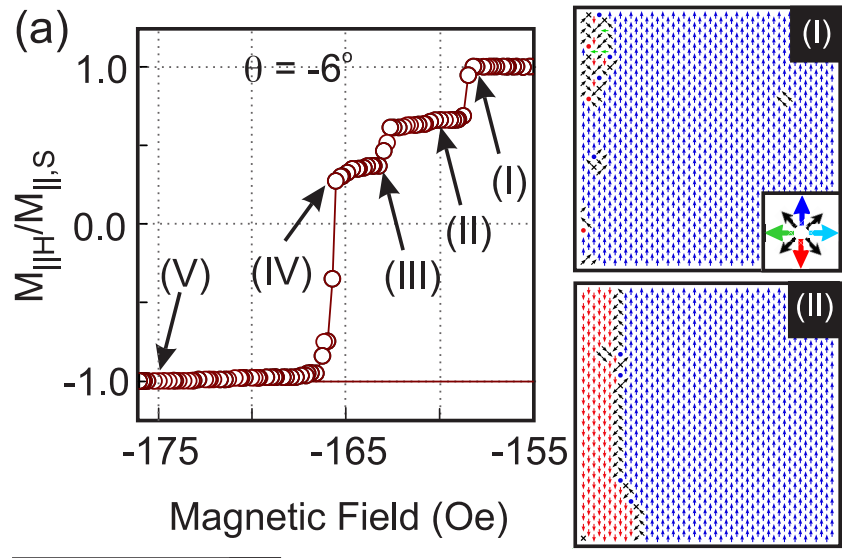

(b)
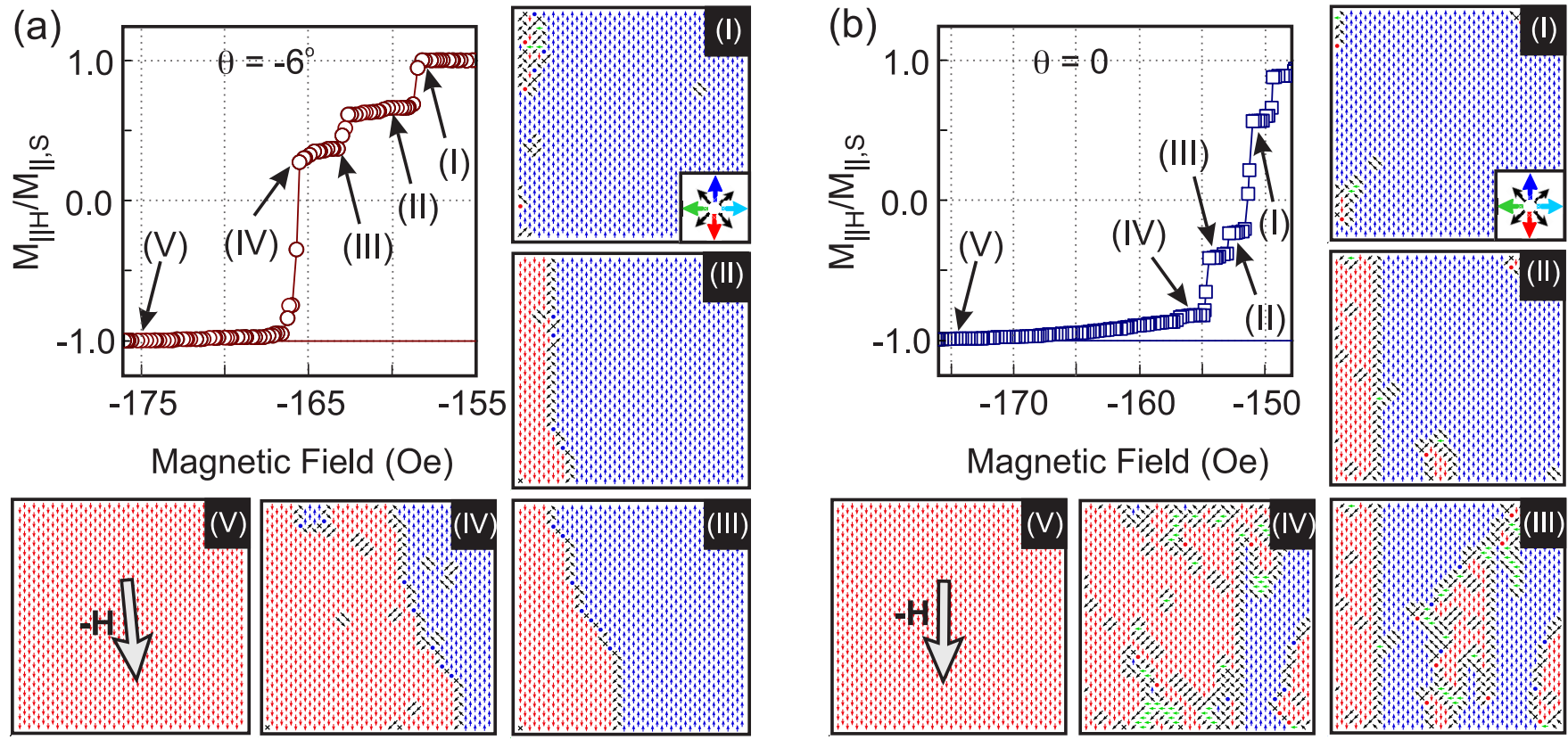

Figure 3. Field-induced domain growth and domain wall patterns in an entire ASI array with applied field angles of $(\mathrm{a}) \theta=-6^{\circ}$ and $(\mathrm{b}) \theta=$ 0 . For both cases, five points $((\mathrm{I})-(\mathrm{V}))$ are marked in the hysteresis loops across the reversal. We also give snapshots of the net moment of the pinwheel units composing the array at each point marked. The unit magnetization orientation is depicted by color-coded arrows, as shown by the arrow color wheel inset in (I). Further information on the net moment and magnetic charge for all possible domain and domain wall unit configurations is provided in Figure S9.

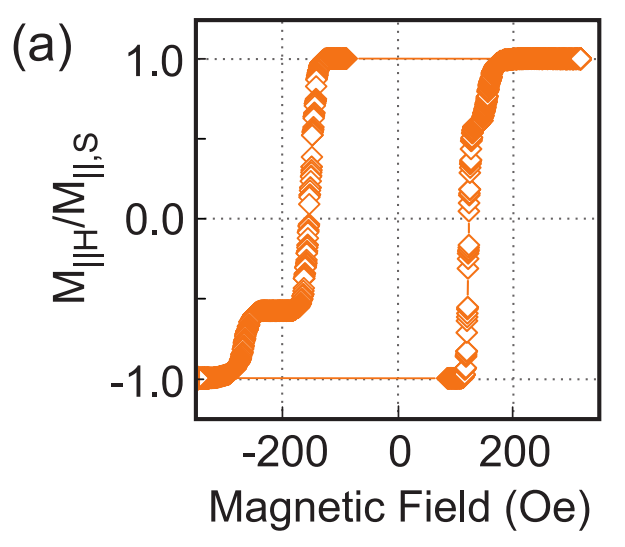

(b)

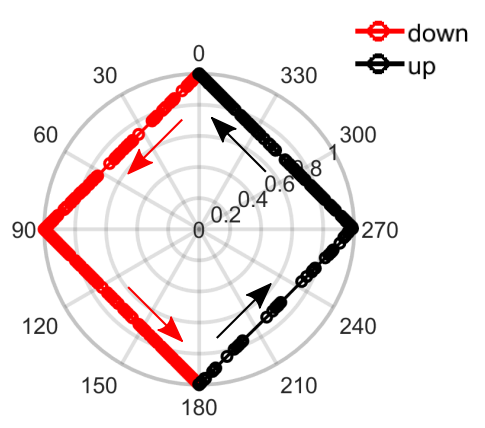

(c)
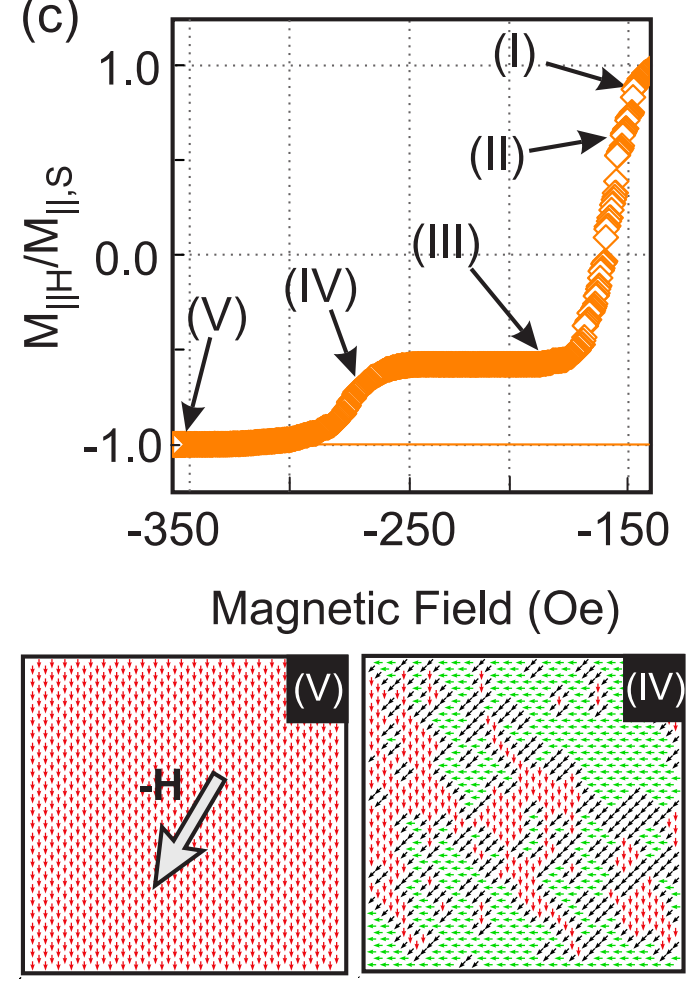
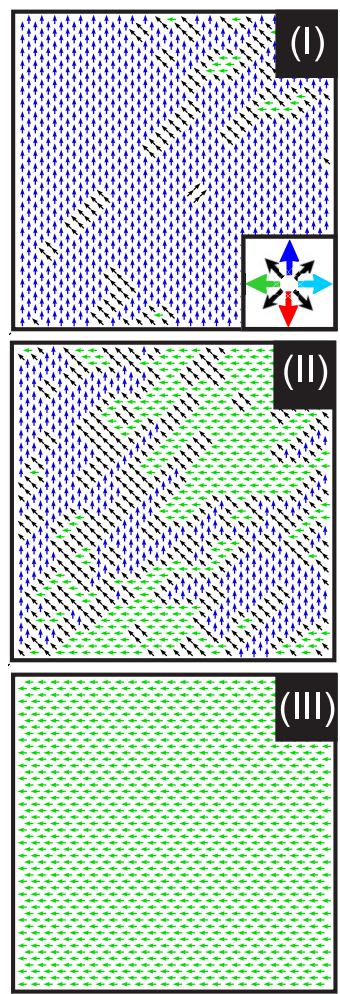

Figure 4. Hysteresis behavior of the (a) net magnetization component parallel to the direction of the externally applied field and (b) the net magnetization with respect to the array edges in polar form, with the field applied at an angle of $30^{\circ}$. (c) Field-induced domain growth and domain wall patterns in an entire ASI array. The unit magnetization orientation is depicted by the color-coded arrows as defined in the inset to part 1 of $(c)$.

the full reversal and in the snapshots of individual island reversals shown in Figure S10). Here, we only focus on the general domain reversal behavior which can be observed from the color contrast. 
(a) $180 \mathrm{~N}$

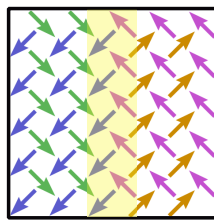

(b) $180 \mathrm{NC}$

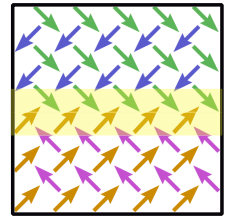

(c) $180 \mathrm{NCD}$

(d) $180 \mathrm{X}$
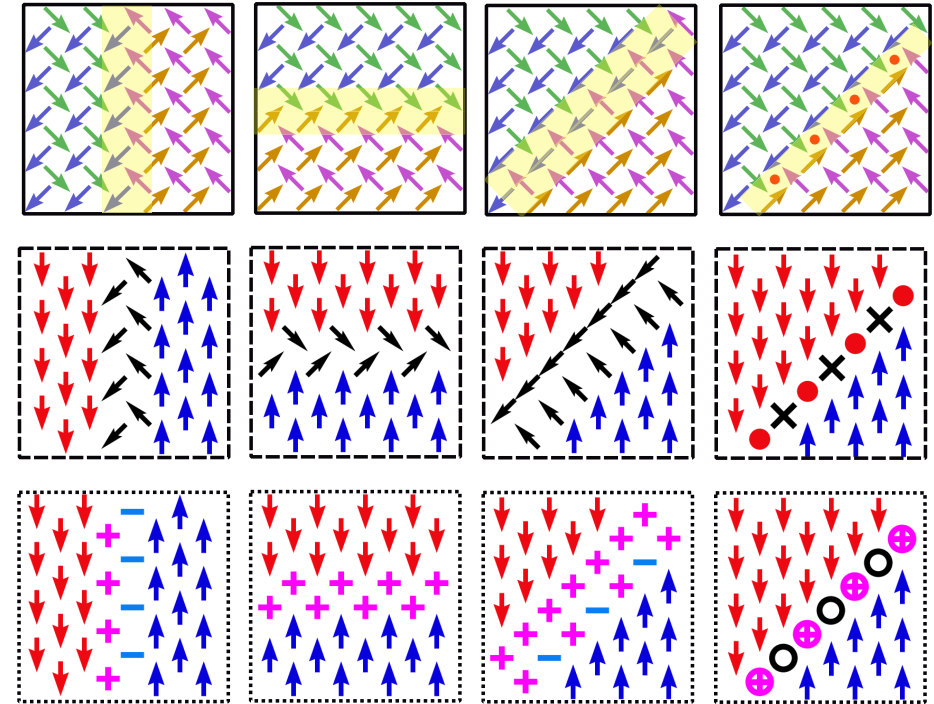

(e) $90 \mathrm{NC}$

(f) $90 \mathrm{~N}$

(g) $90 \mathrm{NCD}$
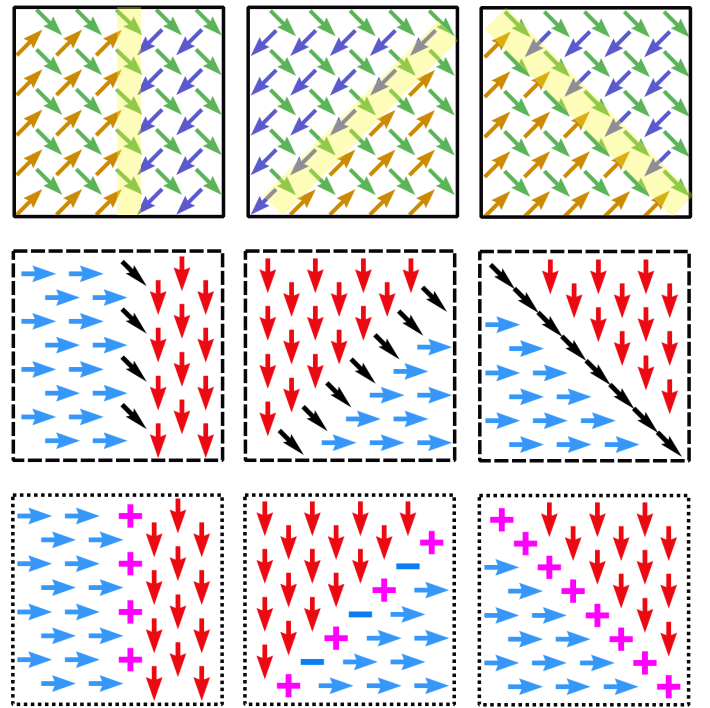

(h)

\begin{tabular}{l|cccc|ccc}
\hline Name & $180 \mathrm{~N}$ & $180 \mathrm{NC}$ & $180 \mathrm{NCD}$ & $180 \mathrm{X}$ & $90 \mathrm{NC}$ & $90 \mathrm{~N}$ & $90 \mathrm{NCD}$ \\
\hline DW type & \multicolumn{7}{c}{$180^{\circ}$} \\
\hline Configurations & 4 & 4 & 4 & 8 & 8 & 4 & 4 \\
Domains align & Antiparallel & $\mathrm{HH}(\mathrm{TT})$ & $\mathrm{HH}(\mathrm{TT})$ & $\mathrm{HH}(\mathrm{TT})$ & 'T'shape & HT(TH) & HH (TT) \\
Unit net charge & 0 & $+2 \mathrm{q}(-2 \mathrm{q})$ & $+2 \mathrm{q}(-2 \mathrm{q})$ & $+2 \mathrm{q}(-2 \mathrm{q})$ & $2 \mathrm{q} /-2 \mathrm{q}$ & 0 & $+2 \mathrm{q}(-2 \mathrm{q})$ \\
Unit net moment & $2 \mathrm{M}$ & $2 \mathrm{M}$ & $2 \mathrm{M}$ & 0 & $\mathrm{M}$ & $\mathrm{M}$ & $\mathrm{M}$ \\
\hline
\end{tabular}

Figure 5. $(\mathrm{a}-\mathrm{d})$ Schematic examples of the possible $180^{\circ} \mathrm{DW}$ configurations in pinwheel ASI containing four categories of domain wall (DW): $180 \mathrm{~N}(\mathrm{a}), 180 \mathrm{NC}(\mathrm{b}), 180 \mathrm{NCD}(\mathrm{c})$, and $180 \mathrm{X}(\mathrm{d}) .(\mathrm{e}-\mathrm{g})$ Schematics of possible $90^{\circ} \mathrm{DW}$ configurations consisting of three types: 90NC (e), 90N (f), and 90NCD (g). The smaller arrows of the configuration at the top, framed by the black solid box, represent the magnetic moments of islands with domain walls highlighted in yellow. The larger arrows in the middle and bottom row images indicate the unit moments of the domains and walls. The bottom images (with dotted frames) show the net charge distributions of the same DWs, where the " \pm " signs reveal the net magnetic charges of Type III units with $\pm 2 q$, the " $\oplus / \ominus$ " symbols indicate the charged Type IV units with $\pm 4 q$, and the open black circles represent the zero-charge Type I units. The net charge of each unit is determined by the dipole magnetic charges of the island using dumbbell model (see Figure S9c). (h) Summary of DW features in which HH, TT, HT, and TH are short for head-tohead, tail-to-tail, head-to-tail, and tail-to-head, respectively.

When the array is saturated (e.g., marked as (V) in Figure 3), a single mesoscopic domain is formed by the so-called Type II units. The Type II unit possesses the largest net moment, as one might expect, and zero net magnetic charge. At small angles of applied field, as shown in Figure 3, reversal starts through a small number of nucleation points, typically located at the edge of the array where the element reversal energy is lower, and progresses by domain growth through domain wall movement perpendicular to the direction of the field. We note that the behavior of magnetization reversal at low-field angles mimics that observed for easy-axis reversal of continuous ferromagnetic films with uniaxial anisotropy. ${ }^{28}$ Interestingly, the reversal appears somewhat more ordered at $\theta=-6^{\circ}$ than at $\theta=0$. This angle offset is consistent with the analysis results of the previous section which showed that the easy anisotropy axis for this array lies at around $-6^{\circ}$.

Reversal at High Applied Field Angles. At higher angles of applied field with respect to the array edge, the reversal process is quite different. This is because, as $\theta$ is increased from 0 to $45^{\circ}$, the easy axis of one sublattice and the hard axis of the other one will become more closely aligned with the field.
Therefore, one sublattice will switch before the other one during a reversal. This gives rise to a "ratcheting" behavior yielding a stepped hysteresis loop as shown in Figure 4a for an applied field angle $\theta=30^{\circ}$ (further details can be seen in the supplemental Video S2 of the full reversal and snapshots of individual island reversal). The coercive field value varies slightly in the positive and negative halves of the $\mathrm{M}-\mathrm{H}$ loop and across all field angles but the variation is generally much smaller than in the $30^{\circ}$ data, as can be seen by comparison to Figure $2 \mathrm{a}-\mathrm{c}$. We attribute these small variations to small differences in the precise magnetization configuration during each reversal. The larger difference in the coercive fields in the $30^{\circ}$ field angle data may be due to a small sample movement during the measurement changing the applied field angle with respect to the array.

At sufficiently high angles of applied field, the large difference in the applied field angle with respect to the easy axes of the two sublattices causes one sublattice to completely reverse before the other one starts. Because the shape anisotropy of an island only allows the moment to align parallel or antiparallel to the long-axis, the array net 
magnetization is constrained to move along $45^{\circ}$ lines. This can be seen for the data in Figure $4 \mathrm{a}$ in the polar plot of the same data in Figure 4b. Starting with the moment pointing up, when the first sublattice reverses, the moment moves from north to west, and when the second sublattice reverses, the net moment changes from west to south, and so on. Thus, the hysteresis loops at high angles of applied field describe a rotated square with a sense of direction that reflects the misalignment angle between the field and the anisotropy axis.

The behavior of the hysteresis loops shown at higher angles of applied field can be translated into a reversal process mediated through a different mechanism to that at low applied field angles. This can be seen in the texture of the magnetization across the reversal shown in the snapshots of the magnetization in Figure 4c. In this case, islands in the sublattice with their easy axes more closely aligned with the field are more likely to reverse first, forming diagonal stripe patterns. Examples of this can be seen in panels (I) and (II) of Figure $4 \mathrm{c}$. As the nanomagnets do not couple strongly to those in adjacent diagonal lines, reversal of the entire array occurs through many nucleation points, creating a spatially inhomogeneous reversal with scattered stripe domains. When one sublattice completely switches (e.g., at point (III) in the hysteresis plot in Figure 4c), the fully magnetized net magnetization lies perpendicular to the initial domain direction. The process then repeats for the other sublattice, to complete the reversal. As a consequence of different reversal mechanisms, the critical field angle marking the transition between the square and stepped loops can be determined from the relative populations of domain walls. This is described in the next section.

Mesoscopic Domain-Wall Topologies. In the reversal processes that were described in the previous section, large domains are seen at low angles of applied field, separated by transition regions, much like domains and domain walls in continuous ferromagnetic films. In pinwheel ASI, the domain walls separate neighboring mesoscopic domains, and each wall type exhibits a discrete macrospin texture. In the reversal regime seen at low applied field angles, the domains are almost entirely formed by Type II units grouped together throughout a reversal. These carry the largest moments which appear in the macroscale as superferromagnetism. Within a domain wall, the macrospin texture is composed by the arrangements of either Type II or a mixture of Type IV and I units. These can be identified by seven classes of domain walls, which are depicted in the columns of Figure $5 \mathrm{a}-\mathrm{g}$, where the top row shows one possibility for a magnetic island configuration, the middle row shows its equivalent unit magnetization, and the bottom row shows the unit charge determined using a

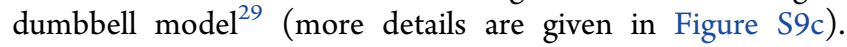
The " \pm " symbols represent Type III units with two positive/ negative net magnetic charges; “ $\oplus$ " “ $\ominus$ ” indicates the positive/negative Type IV units possessing four charges; and " $O$ " are the uncharged Type I units.

Each class of domain wall has multiple element configurations. For example, in Figure 5a, two neighboring domains possess two possible alignments, where the net magnetizations are antiparallel, and the domain wall has two possible magnetization directions (pointing to the left or right). As a consequence, this domain wall has four possible magnetization configurations. The number of all possible wall configurations is indicated in the third row of the table in Figure 5h (other possible wall configurations are given in Figure S11). The seven domain walls can be categorized by the angle between magnetization orientations of the adjacent domains into $180^{\circ}$ or $90^{\circ}$ domain walls. All walls can be further categorized by the alignment of the adjacent domains: either antiparallel, head-tohead $(\mathrm{HH})$, tail-to-tail (TT), or head-to-tail (HT) and vice vers $a$ and by the net charge and moment of a unit, as indicated by the remaining rows of the table.

Drawing from continuous film ferromagnets, we designate all walls in pinwheel ASI by the angle between adjacent domains followed by the minimum number of letters denoting the wall type. For the $180^{\circ}$ walls (Figure 5a-d), the walls are Néel $(\mathrm{N})$, charged Néel (NC), diagonal charged Neel (NCD) and diagonal cross-tie walls $(\mathrm{X})$. The $180 \mathrm{~N}$ walls are analogues of a classical Néel wall, ${ }^{30,31}$ which is uncharged, and the $180 \mathrm{X}$ wall resembles a cross-tie wall ${ }^{32}$ formed by alternating Type IV and Type I units. The $180 \mathrm{NC}$ and $180 \mathrm{NCD}$ walls are charged walls not seen in continuous ferromagnetic films which lack the reduced degrees of freedom of our pinwheel lattice. We note, however, that analogous domain wall configurations are commonly observed in highly anisotropic continuous structures such as nanowires. 33,34

The polarity of charged walls depends on the magnetization orientation of the adjacent domains. For example, the domain wall carries positive or negative net unit charge when the magnetization directions of neighboring domains are head-tohead or tail-to-tail, respectively. This is analogous to the characteristic signatures of charged walls in ferroelectric materials $^{35,36}$ in which walls carry polarized electrostatic charges.

All $90^{\circ}$ walls (Figure $5 \mathrm{e}-\mathrm{g}$ ) separate domains in which the magnetization directions lie at right angles to one another, and all exhibit Néel rotation. Following the $180^{\circ}$ wall naming system, we denote these as $90 \mathrm{NC}, 90 \mathrm{~N}$, and $90 \mathrm{NCD}$. The uncharged $90 \mathrm{~N}$ wall is analogous to a classical Néel wall. As in the $180^{\circ}$ walls, charged Neel walls exist in the $90^{\circ}$ wall types which are not found in natural ferromagnets due to the energetically unfavorable head-to-head (see Figure 5) or tailto-tail (see Figure S11) alignment. The charge ordering of this wall type is also found to be dependent on the magnetization orientation of their adjacent domains. These peculiar properties of specific charge ordering in pinwheel ASI are the direct result of the high anisotropy within a system of discrete magnetization.

At the level of individual islands, the fundamental difference between the formation of $180^{\circ}$ and $90^{\circ}$ walls arises from individual moment reversals of the two sublattices, as illustrated by the top row images of Figure $5 \mathrm{a}-\mathrm{g}$. When one goes through the domain wall interface of a $180^{\circ}$ wall, the spins in both sublattices reverse simultaneously, whereas for a $90^{\circ}$ wall, only spins in one of the sublattices flip. Thus, it is impossible to form the $90 \mathrm{X}$ wall equivalent to the $180 \mathrm{X}$ wall. This different formation mechanism between two categories of walls can be used to map the transition between the reversal regimes of pinwheel ASI, from ferromagnetic-like ordering at low angles of applied field to the spatially inhomogeneous reversal at higher angles of applied field. As the applied field angle increases from zero, one sublattice easy axis becomes more aligned with the field, while that of the other lies at a higher angle to the field, and thus, the two sublattices are no longer coupled, and a transition from $180^{\circ}$ walls to $90^{\circ}$ ones should occur.

To characterize the extent of the ordered and the spatially inhomogeneous reversal regimes, we examine the domain wall 
population statistics across an $\mathrm{M}-\mathrm{H}$ loop as a function of the applied field angle by counting the number of times a domain wall "motif" appears at each applied field strength.

This result is shown in Figure $6 \mathrm{a}, \mathrm{b}$ for $180^{\circ}$ and $90^{\circ}$ walls, respectively. The domain wall motifs represent the smallest

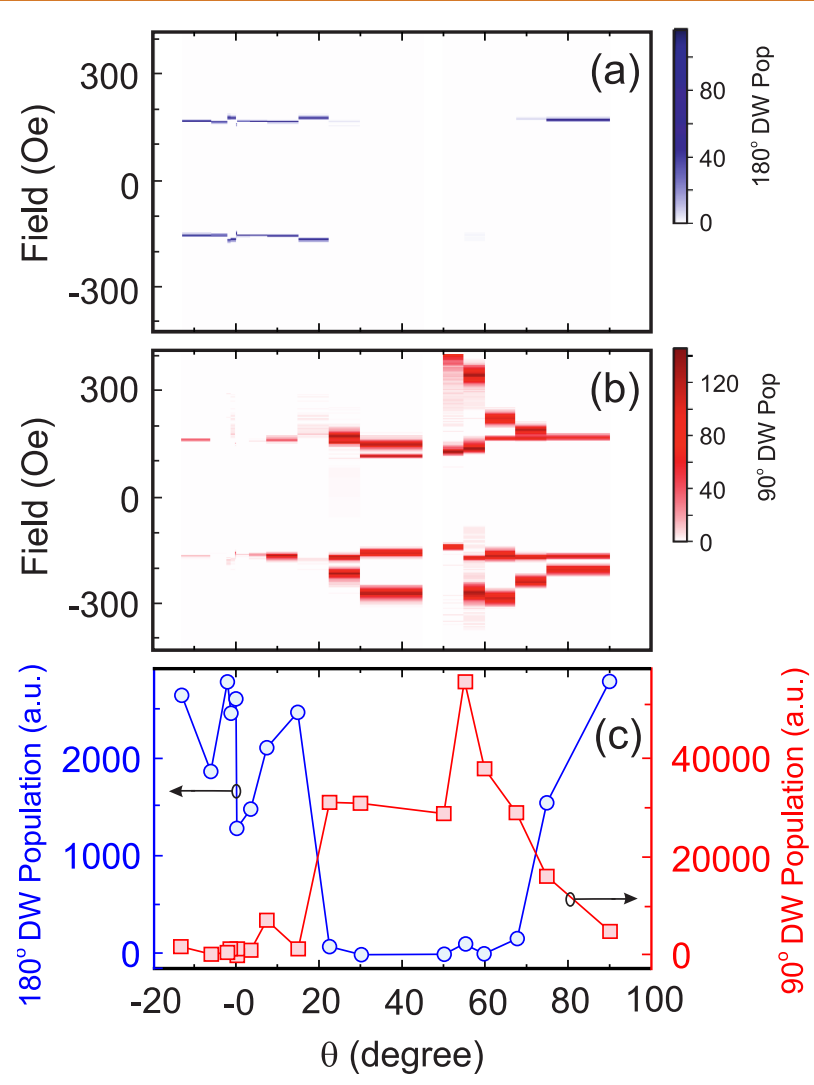

Figure 6. (a) $180^{\circ}$ and (b) $90^{\circ} \mathrm{DW}$ total populations as a function of the magnetic field strength in a full hysteresis loop as a function of the applied field angle, $\theta$. (c) Field-angle dependence of the accumulative $180^{\circ}$ and $90^{\circ} \mathrm{DW}$ populations across the field sweep of the hysteresis loops shown in (a). The domain wall motifs are defined in Figure S12.

cluster of spins that define each wall (see sketch in Figure S12), and these are composed by multiple pinwheel units such as those discussed so far in the context of unit net moment (see insets in Figure 1). From Figure 6a,b we can infer that the reversal process at low angles happens mostly though the formation of $180^{\circ}$ walls and it extends from at least $-13^{\circ}$ (the minimum angle measured) to $\sim 20^{\circ}$. The $180^{\circ}$ walls mostly disappear at higher applied field angles (from $\sim 20^{\circ}$ to $\sim 70^{\circ}$ ), giving way to the formation of $90^{\circ}$ walls (examples of all individual wall motif populations are given in the Figure S13). Reversal in the latter, spatially inhomogeneous regime clearly happens in a two-step process where one sublattice reverses before the other, as shown in Figure 4. The minimum absolute coercive field does not change dramatically across all angles and regimes because the shape anisotropy of the individual island is the main determinant of this property and of the anisotropic nature of the array, with inter-island coupling having a smaller but important effect. To visualize the regime angular dependence more easily, it is useful to build a domain wall population count for the full $\mathrm{M}-\mathrm{H}$ loop as a function of angle. In Figure $6 \mathrm{c}$, we show the sum of all $180^{\circ}$ and all $90^{\circ}$ wall units for each full loop at each applied field angle from $-13^{\circ}$ to $90^{\circ}$, and indeed, we see a preferential behavior of these domain walls for the same range of angles seen in Figure $6 \mathrm{a}, \mathrm{b}$ before the integration across the $\mathrm{M}-\mathrm{H}$ loop.

Figure 6 shows the existence of the two main reversal regimes (it is worth noting that information on the continuity of the specific domain walls that form in these regimes exists in the source data). At low angles of applied field, reversal is typically mediated by a low number of mesoscopic domain walls. With the field aligned at $-6^{\circ}$ (see Figure $3 a$ ), one main mesoscopic domain wall exists and is comprised by connected $180 \mathrm{~N}$ and $180 \mathrm{X}$ walls, accompanied by a small number of $180 \mathrm{NCD}$ walls (see Figure S13b for population statistics). As the wall propagates, the locations of the $180 \mathrm{~N}$ and $180 \mathrm{X}$ walls change, depending on the angle of their adjacent domains, but the wall remains connected throughout the vast majority of the reversal. The complete reversal can be seen in detail in Video S1. At higher angles of applied field, as one sublattice reverses, type III units form with a diagonal moment. These can form $90 \mathrm{~N}$ and $90 \mathrm{NC}$ domain walls or regions of type III units that may be considered domains in themselves (examples of these can be seen in Figure 4c and Video S2 for the applied field angle of $30^{\circ}$ ). Unlike in the low-angle field regime, due to the more inhomogeneous reversal, it is hard to follow the specific domain wall propagation here, but we observe that the $90 \mathrm{~N}$ wall is more continuous than are the $90 \mathrm{NC}$ ones during the reversal. This may be seen by observing that the domain walls mainly lie at $45^{\circ}$ to the array edge, giving rise to $90 \mathrm{~N}$ walls, while the $90 \mathrm{NC}$ walls are somewhat more scattered across the array.

\section{DISCUSSION AND CONCLUSIONS}

Pinwheel ASI provides an example of how a simple geometry modification can dramatically affect the magnetic properties of a spin ice array. Here, we have shown experimentally the emergence of superferromagnetism in this structure.

We expect array edges to influence aspects of the magnetization such as array anisotropy and that the extended nature of the island may also have some effect. For instance, recent theoretical work on the pinwheel geometry has shown that the details of the thermal ground state depend on the array edges. $^{22}$ In order to probe this experimentally, preliminary measurements comparing magnetization processes in diamond pinwheel arrays with different edge cuts have been made. The results are suggestive that there may be an effective magnetic anisotropy axis dependence on array edge geometry (see Figure S7). Definitive conclusions on this aspect require a more detailed and extensive experimental study that goes beyond the scope of the present work.

The reversal process is strongly affected by the direction of the field with respect to the array edges. In pinwheel ASI, the dimensionality of magnetic avalanches in the reversal process is determined by the field angle $\theta$. In the low-field-angle regime ( $\lesssim 20^{\circ}$ from array edges), the magnetic state is analogous to ferromagnetic order. Reversal of the mesoscopic domains in this regime is through two-dimensional avalanches of macrospins (see Figure S10), while at applied field angles approaching $45^{\circ}$ the magnetization is disordered and reversal is through the formation of one-dimensional (1-D) stripes. The low applied field angle behavior is the opposite of the magnetization processes in square ASI, where 1-D monopolelike defects and dirac-like strings form, ${ }^{5,11,14,15,37,38}$ and is a direct result of modification of the inter-island coupling. In square ASI, the strongest coupling is with the nearest 
neighbors, and the coupling strength falls off monotonically with increasing distance. ${ }^{39}$ By rotating each island in square ASI by $45^{\circ}$, the nearest-neighbor coupling that is dominant in square ASI is greatly reduced, and the dipolar coupling strength increases with distance, peaking at the fourth nearest neighbor. $^{22}$

The pinwheel ASI system is prone to the formation of domain walls analogous to those seen in continuous film natural ferromagnetic materials, such as Néel and cross-tie walls. But due to the lack of the exchange interaction, the mesoscopic walls here are not topologically protected. On the other hand, weak inter-island coupling in this pinwheel ASI system allows for the formation of Néel walls under a lowangle magnetic field with underlying ferromagnetic features, which have not been observed in other ASI systems. Other intriguing domain walls types can also be seen. These have specific charge ordering and net moments due to the high anisotropy and constrained degrees of freedom of the system. Furthermore, we have shown that by simply changing the orientation of the externally applied field with respect to the array edges, it is possible to completely modify the nature of the domain wall configurations and the field evolution. This property of pinwheel ASI could be used to effectively tune devices based on magnetotransport phenomena such as the recently suggested Hall circuits. ${ }^{40}$

Lastly, the work reported here has concentrated on one particular array geometry. The key driving force behind the interest in other ASI systems has been the tunability of key magnetic properties through the geometrical design. We expect this also to be true for the superferromagnetic pinwheel ASI, where the coupling parameters can be varied so that different phases can be achieved or controlled. Our results show that this structure presents an interesting model system for experimental exploration of fundamental magnetization processes such as magnetic interfaces, exchange bias phenomena, and spin wave propagation. For example, the array geometry may be tailored in such a way as to extend the Ising-like domain walls reported here to spread over several elements, potentially leading to controlling over the mesoscopic wall formation and propagation.

\section{METHODS}

Sample Fabrication. The permalloy $\left(\mathrm{Ni}_{80} \mathrm{Fe}_{20}\right)$ ASI arrays were patterned on electron-transparent silicon nitride $\left(\mathrm{Si}_{3} \mathrm{~N}_{4}\right)$ membranes using electron beam lithography and lift-off metallization. The $\mathrm{Si}_{3} \mathrm{~N}_{4}$ membranes were spin coated with ZEP520A:anisole (1:1) with film thickness $\approx 140 \mathrm{~nm}$ at $4 \mathrm{krpm}$ for $40 \mathrm{~s}$ and baked at $180{ }^{\circ} \mathrm{C}$ for $180 \mathrm{~s}$. The spin ice arrays were then defined using electron beam lithography using an electron dose of $343 \mu \mathrm{C} / \mathrm{cm}^{2}$. The pattern was developed for $70 \mathrm{~s}$ in N50 solution. $10 \mathrm{~nm} \mathrm{Ni}{ }_{80} \mathrm{Fe}_{20}$ was evaporated into the pattern and lifted off in microposit remover 1165 at $70{ }^{\circ} \mathrm{C}$. An aluminum capping layer was used to prevent the sample from oxidizing. Selected area electron diffraction and dark-field imaging show the permalloy film is polycrystalline with grain sizes less than $\sim 10 \mathrm{~nm}$ in width (further details are supplied in Figure S1).

LTEM Measurement. All experimental results in this work are from Fresnel imaging of ASI arrays in a JEOL ARM $200 \mathrm{cF}$ equipped with a cold field emission gun and operated at $200 \mathrm{kV}$. The beam spot size was 2 , the emission current was $14 \mu \mathrm{A}$, and a $70 \mu \mathrm{m}$ condenser aperture was used. The standard JEOL single tilt and rotate TEM sample holder was employed to tilt and rotate the sample, while a Gatan Orius SC1000A CCD camera was used to take Fresnel imaging videos.

In Fresnel imaging, the Lorentz force deflection of an electronbeam by the integrated induction produced by each island creates bright and dark edges along the long axis in a defocused image from which the direction of magnetization can be inferred. ${ }^{41,42}$ The defocus in the experiments was $5 \mathrm{~mm}$. An example of a Fresnel image of saturated pinwheel ASI is shown in the lower left part of Figure 1, where the arrows in the bottom inset indicate the magnetization of each of four islands composing a pinwheel unit.

In LTEM mode, the objective lens can be entirely switched off to create a low-field environment for the sample, and a objective mini lens below this lens is used to act as an imaging lens. A magnetic field along the optic axis, $H_{\mathrm{ob}}$, is created by applying a small current to the main objective lens. The magnetic field strength was determined from the current by calibrating the field-current relationship of the lens with a Hall probe. The in-plane component of the magnetic field was varied by tilting the sample between $\pm 25^{\circ}$ and $\mp 25^{\circ}$ in the fixed 700 Oe magnetic field of the objective lens, $H_{\mathrm{obj}}$, as shown in the schematic in Figure S14. The strength of the in-plane field can be obtained by $H_{\text {in-plane }}=H_{\text {obj }} \times \sin \alpha$, where $\alpha$ is the tilting angle of the sample holder. A Matlab-controlled tilting GUI was developed to automatically tilt the sample holder at a constant rate. The angle of inplane magnetic field with respect to the sample was changed by rotating the specimen in the plane of the sample using a JEOL single tilt and rotate sample holder. A $10 \mathrm{fps}$ video was recorded to track the evolution of the magnetization. The videos were then processed to extract the net magnetization direction of each island using the magnetic contrast discussed in the manuscript, assuming a singledomain exists and the island acts as a macrospin. Details of the image processing methodology are given in Supporting Information. The raw Fresnel images and the corresponding processed Ising moment configurations of the panels $(\mathrm{I})-(\mathrm{V})$ in Figures 3 and 4 are also provided in Figures S3-S5.

\section{ASSOCIATED CONTENT}

\section{Supporting Information}

The Supporting Information is available free of charge on the ACS Publications website at DOI: 10.1021/acsnano.8b08884.

Material characterization results, details of the Fresnel image processing methodology, calculations of an Ising hyteresis loop based on field protocols, figures showing anisotropy axis orientations of two types pinwheel ASI arrays with symmetric and asymmetric boundaries, introduction to the transform from square vertices to pinwheel units, figures of domain and domain wall formations (PDF)

Video S1: Magnetization reversal of the pinwheel ASI array at the low-field angle of $-6^{\circ}$ (AVI)

Video S2: Magnetization reversal of the pinwheel ASI array at the high-field angle of $30^{\circ}$ (AVI)

\section{AUTHOR INFORMATION}

\section{Corresponding Authors}

*E-mail: Gary.Paterson@glasgow.ac.uk.

*E-mail: Stephen.McVitie@glasgow.ac.uk.

*E-mail: Robert.Stamps@umanitoba.ca.

ORCID $\odot$

Yue Li: 0000-0001-9171-0355

Gary W. Paterson: 0000-0002-4680-048X

Rair Macêdo: 0000-0003-3358-798X

Notes

The authors declare no competing financial interest.

\section{ACKNOWLEDGMENTS}

This work was supported by the Engineering and Physical Sciences Research Council (EPSRC grant nos. EP/L002922/1 and $\mathrm{EP} / \mathrm{L} 00285 \mathrm{X} / \mathrm{1}$ ) and the University of Glasgow. Y. Li was 
funded by the China Scholarship Council. G. W. Paterson, S. McVitie, and D. A. MacLaren received partial support from EPSRC grant no. EP/M024423/1. G. M. Macauley received support from the Carnegie Trust for the Universities of Scotland. R. Macêdo was supported by the Leverhulme trust. R. L. Stamps acknowledges the support of the Natural Sciences and Engineering Research Council of Canada (NSERC) - R. L. Stamps a été financée par le Conseil de recherches en sciences naturelles et en génie du Canada (CRSNG). F. S. Nascimento has benefited from discussions with A. R. Pereira. Original data files are available at http://dx.doi.org/10.5525/gla. researchdata.719.

\section{REFERENCES}

(1) Wang, R. F.; Nisoli, C.; Freitas, R. S.; Li, J.; McConville, W.; Cooley, B. J.; Lund, M. S.; Samarth, N.; Leighton, C.; Crespi, V. H.; Schiffer, P. Artificial 'Spin Ice' in a Geometrically Frustrated Lattice of Nanoscale Ferromagnetic Islands. Nature 2006, 439, 303-306.

(2) Perrin, Y.; Canals, B.; Rougemaille, N. Extensive Degeneracy, Coulomb Phase and Magnetic Monopoles in Artificial Square Ice. Nature 2016, 540, 410-413.

(3) Wang, Y. L.; Xiao, Z. L.; Snezhko, A.; Xu, J.; Ocola, L. E.; Divan, R.; Pearson, J. E.; Crabtree, G. W.; Kwok, W. K. Rewritable Artificial Magnetic Charge Ice. Science 2016, 352, 962-966.

(4) Zeissler, K.; Walton, S. K.; Ladak, S.; Read, D. E.; Tyliszczak, T.; Cohen, L. F.; Branford, W. R. The Non-random Walk of Chiral Magnetic Charge Carriers in Artificial Spin Ice. Sci. Rep. 2013, 3, 1252 .

(5) Mengotti, E.; Heyderman, L. J.; Rodríguez, A. F.; Nolting, F.; Hügli, R. V.; Braun, H.-B. Real-Space Observation of Emergent Magnetic Monopoles and Associated Dirac Strings in Artificial Kagome Spin Ice. Nat. Phys. 2011, 7, 68-74.

(6) Drisko, J.; Marsh, T.; Cumings, J. Topological Frustration of Artificial Spin Ice. Nat. Commun. 2017, 8, 14009.

(7) Zhang, S.; Gilbert, I.; Nisoli, C.; Chern, G.-W.; Erickson, M. J.; O’Brien, L.; Leighton, C.; Lammert, P. E.; Crespi, V. H.; Schiffer, P. Crystallites of Magnetic Charges in Artificial Spin Ice. Nature 2013, 500, 553-557.

(8) Gilbert, I.; Lao, Y.; Carrasquillo, I.; O’Brien, L.; Watts, J. D.; Manno, M.; Leighton, C.; Scholl, A.; Nisoli, C.; Schiffer, P. Emergent Reduced Dimensionality by Vertex Frustration in Artificial Spin Ice. Nat. Phys. 2016, 12, 162-165.

(9) Gilbert, I.; Chern, G.-W.; Zhang, S.; O’Brien, L.; Fore, B.; Nisoli, C.; Schiffer, P. Emergent Ice Rule and Magnetic Charge Screening from Vertex Frustration in Artificial Spin Ice. Nat. Phys. 2014, 10, 670-675.

(10) Lammert, P. E.; Ke, X.; Li, J.; Nisoli, C.; Garand, D. M.; Crespi, V. H.; Schiffer, P. Direct Entropy Determination and Application to Artificial Spin Ice. Nat. Phys. 2010, 6, 786-789.

(11) Silva, R. C.; Lopes, R. J. C.; Mól, L. A. S.; Moura-Melo, W. A.; Wysin, G. M.; Pereira, A. R. Nambu Monopoles Interacting with Lattice Defects in a Two-Dimensional Artificial Square Spin Ice. Phys. Rev. B: Condens. Matter Mater. Phys. 2013, 87, 014414.

(12) Ladak, S.; Read, D. E.; Branford, W. R.; Cohen, L. F. Direct Observation and Control of Magnetic Monopole Defects in an Artificial Spin-Ice Material. New J. Phys. 2011, 13, 063032.

(13) Branford, W. R.; Ladak, S.; Read, D. E.; Zeissler, K.; Cohen, L. F. Emerging Chirality in Artificial Spin Ice. Science 2012, 335, 15971600.

(14) Hügli, R. V.; Duff, G.; O’Conchuir, B.; Mengotti, E.; Rodríguez, A. F.; Nolting, F.; Heyderman, L. J.; Braun, H. B. Artificial Kagome Spin Ice: Dimensional Reduction, Avalanche Control and Emergent Magnetic Monopoles. Philos. Trans. R. Soc., A 2012, 370, 5767-5782.

(15) Gliga, S.; Kákay, A.; Hertel, R.; Heinonen, O. G. Spectral Analysis of Topological Defects in an Artificial Spin-Ice Lattice. Phys. Rev. Lett. 2013, 110, 117205.
(16) Shi, D.; Budrikis, Z.; Stein, A.; Morley, S. A.; Olmsted, P. D.; Burnell, G.; Marrows, C. H. Frustration and Thermalization in an Artificial Magnetic Quasicrystal. Nat. Phys. 2018, 14, 309-314.

(17) Morgan, J. P.; Stein, A.; Langridge, S.; Marrows, C. H. Thermal Ground-State Ordering and Elementary Excitations in Artificial Magnetic Square Ice. Nat. Phys. 2011, 7, 75-79.

(18) Farhan, A.; Derlet, P. M.; Kleibert, A.; Balan, A.; Chopdekar, R. V.; Wyss, M.; Perron, J.; Scholl, A.; Nolting, F.; Heyderman, L. J. Direct Observation of Thermal Relaxation in Artificial Spin Ice. Phys. Rev. Lett. 2013, 111, 057204.

(19) Pauling, L. The Structure and Entropy of Ice and of Other Crystals with Some Randomness of Atomic Arrangement. J. Am. Chem. Soc. 1935, 57, 2680-2684.

(20) Morrison, M. J.; Nelson, T. R.; Nisoli, C. Unhappy Vertices in Artificial Spin Ice: New Degeneracies from Vertex Frustration. New J. Phys. 2013, 15, 045009.

(21) Gliga, S.; Hrkac, G.; Donnelly, C.; Bchi, J.; Kleibert, A.; Cui, J.; Farhan, A.; Kirk, E.; Chopdekar, R. V.; Masaki, Y.; Bingham, N. S.; Scholl, A.; Stamps, R. L.; Heyderman, L. J. Emergent Dynamic Chirality in a Thermally Driven Artificial Spin Ratchet. Nat. Mater. 2017, 16, 1106-1111.

(22) Macêdo, R.; Macauley, G. M.; Nascimento, F. S.; Stamps, R. L. Apparent Ferromagnetism in the Pinwheel Artificial Spin Ice. Phys. Rev. B: Condens. Matter Mater. Phys. 2018, 98, 014437.

(23) McVitie, S.; McGrouther, D.; McFadzean, S.; MacLaren, D. A.; O'Shea, K. J.; Benitez, M. J. Aberration Corrected Lorentz Scanning Transmission Electron Microscopy. Ultramicroscopy 2015, 152, 5762

(24) Bedanta, S.; Eimüller, T.; Kleemann, W.; Rhensius, J.; Stromberg, F.; Amaladass, E.; Cardoso, S.; Freitas, P. P. Overcoming the Dipolar Disorder in Dense CoFe Nanoparticle Ensembles: Superferromagnetism. Phys. Rev. Lett. 2007, 98, 176601.

(25) Morgan, J. P.; Stein, A.; Langridge, S.; Marrows, C. H. Magnetic Reversal of an Artificial Square Ice: Dipolar Correlation and Charge Ordering. New J. Phys. 2011, 13, 105002.

(26) Morgan, J. P.; Bellew, A.; Stein, A.; Langridge, S.; Marrows, C. H. Linear Field Demagnetization of Artificial Magnetic Square Ice. Front. Phys. 2013, 1, 28.

(27) Budrikis, Z.; Morgan, J. P.; Akerman, J.; Stein, A.; Politi, P.; Langridge, S.; Marrows, C. H.; Stamps, R. L. Disorder Strength and Field-Driven Ground State Domain Formation in Artificial Spin Ice: Experiment, Simulation. Phys. Rev. Lett. 2012, 109, 037203.

(28) Li, Y.; Xu, K.; Hu, S.; Suter, J.; Schreiber, D. K.; Ramuhalli, P.; Johnson, B. R.; McCloy, J. Computational and Experimental Investigations of Magnetic Domain Structures in Patterned Magnetic Thin Films. J. Phys. D: Appl. Phys. 2015, 48, 305001.

(29) Castelnovo, C.; Moessner, R.; Sondhi, S. L. Magnetic Monopoles in Spin Ice. Nature 2008, 451, 42-45.

(30) Middelhoek, S. Domain Walls in Thin Ni-Fe Films. J. Appl. Phys. 1963, 34, 1054-1059.

(31) Lee, D.; Behera, R. K.; Wu, P.; Xu, H.; Li, Y. L.; Sinnott, S. B.; Phillpot, S. R.; Chen, L. Q.; Gopalan, V. Mixed Bloch-Néel-Ising Character of $180^{\circ}$ Ferroelectric Domain Walls. Phys. Rev. B: Condens. Matter Mater. Phys. 2009, 80, No. 060102.

(32) Löhndorf, M.; Wadas, A.; Van Den Berg, H. A. M.; Wiesendanger, R. Structure of Cross-Tie Wall in Thin Co Films Resolved by Magnetic Force Microscopy. Appl. Phys. Lett. 1996, 68, 3635-3637.

(33) Petit, D.; Zeng, H. T.; Sampaio, J.; Lewis, E.; O’Brien, L.; Jausovec, A.-V.; Read, D.; Cowburn, R. P.; O’Shea, K. J.; McVitie, S.; Chapman, J. N. Magnetic Imaging of the Pinning Mechanism of Asymmetric Transverse Domain Walls in Ferromagnetic Nanowires. Appl. Phys. Lett. 2010, 97, 233102.

(34) O’Shea, K. J.; McVitie, S.; Chapman, J. N.; Weaver, J. M. R. Direct Observation of Changes to Domain Wall Structures in Magnetic Nanowires of Varying Width. Appl. Phys. Lett. 2008, 93, 202505.

(35) Meier, D. Functional Domain Walls in Multiferroics. J. Phys.: Condens. Matter 2015, 27, 463003. 
(36) Bednyakov, P. S.; Sluka, T.; Tagantsev, A. K.; Damjanovic, D.; Setter, N. Formation of Charged Ferroelectric Domain Walls with Controlled Periodicity. Sci. Rep. 2015, 5, 15819.

(37) Pollard, S. D.; Volkov, V.; Zhu, Y. Propagation of Magnetic Charge Monopoles and Dirac Flux Strings in an Artificial Spin-Ice Lattice. Phys. Rev. B: Condens. Matter Mater. Phys. 2012, 85, 180402.

(38) Gilbert, I.; Chern, G.-W.; Fore, B.; Lao, Y.; Zhang, S.; Nisoli, C.; Schiffer, P. Direct Visualization of Memory Effects in Artificial Spin Ice. Phys. Rev. B: Condens. Matter Mater. Phys. 2015, 92, 104417. (39) Morley, S. A.; Riley, S. T.; Porro, J.-M.; Rosamond, M. C.; Linfield, E. H.; Cunningham, J. E.; Langridge, S.; Marrows, C. H. Effect of FePd Alloy Composition on the Dynamics of Artificial Spin Ice. Sci. Rep. 2018, 8, 4750.

(40) Chern, G.-W. Magnetotransport in Artificial Kagome Spin Ice. Phys. Rev. Appl. 2017, 8, 064006.

(41) Williams, D. B.; Carter, C. B. In Transmission Electron Microscopy; Plenum Press: New York, 2009; p 398.

(42) Qi, Y.; Brintlinger, T.; Cumings, J. Direct Observation of the Ice Rule in an Artificial Kagome Spin Ice. Phys. Rev. B: Condens. Matter Mater. Phys. 2008, 77, 094418. 